\title{
ARTICLE \\ Roust Power System Stabilizer Design Using Kharitonov's Theorem: A Case Study
}

\section{Hisham Soliman* Mohammed Albadi Hamood Al-Sheriyani Hadhifa Al-Azakawi Ali Al- Qutaiti}

Department of Electrical and Computer Engineering, Sultan Qaboos University, Muscat, Oman

\begin{tabular}{ll}
\hline ARTICLE INFO & ABSTRACT \\
$\begin{array}{l}\text { Article history } \\
\text { Received: } 9 \text { September } 2019\end{array}$ & $\begin{array}{l}\text { This paper proposes a robust power system stabilizer(PSS) for a steam syn- } \\
\text { chronous generator in Barka II power station. The PSS should be capable of } \\
\text { damping small-disturbance oscillations (inherently existing in power sys- } \\
\text { Accepted: } 27 \text { September } 2019\end{array}$ \\
tems due to e.g. load changes, lines switching...etc.) within a certain settling \\
time for different load conditions. Also, the proposed PSS must have the \\
conventional structure and its parameters must not be violated. To achieve \\
this goal, robust control provides many advantages. The suggested control- \\
Keywords: & $\begin{array}{l}\text { ler is tuned by the Kharitonov's theorem and uses the standard structure } \\
\text { employed in industry. The problem is cast into a nonlinear constrained opti- } \\
\text { Power system stabilizer }\end{array}$ \\
mobust control & $\begin{array}{l}\text { mization problem to achieve the desired settling time without violating the } \\
\text { practical values of the controller parameters. Performance of the robust PSS } \\
\text { is evaluated by several simulations in the presence of system uncertainty } \\
\text { Kharitonov's theorem }\end{array}$ \\
due to load changes.
\end{tabular}

\section{Abbreviations}

AVR: Automatic voltage regulator, CPSS: Conventional power system stabilizer, LTI: Linear time-invariant systems, PSS: Power system stabilizer

\section{Nomenclature}

All units are in per unit (p.u) unless otherwise stated.

$\triangle$ : deviation

$\delta$ : Torque angle of machine, rad.

$\omega:$ Speed .

$x_{d}^{\prime}$ : Generator direct-axis transient reactance.

$x_{d}, x_{q}$ : Direct and quadrature-axis synchronous reactance, respectively.

$I_{d}, I_{q}$ :D-axis, Q-axis armature and field winding cur- rents, respectively.

$P, Q$ :Real and reactive power loading, respectively.

$V:$ Terminal voltage of the generator.

$V_{\infty}$ : Infinite bus voltage.

$x_{e}$ : Transmission line reactance.

$H$ : machine inertia constant, sec

$M:$ machine momentum $=2 H$, sec.

$E_{q}^{\prime}$ : The quadrature-axis transient voltage of machine.

$E_{f}$ : The field voltage of machine .

$T_{d 0}^{\prime}$ : D-axis open circuit field time constant, sec.

$K_{E}$ : Gain of the excitation system.

$T_{E}$ : Time constant of the excitation system, sec.

$x$ : The vector of the state variables.

$u$ : The vector of input variables.

$A, B, C$ : State, input, and output matrices, respectively

*Corresponding Author:

Hisham Soliman,

Department of Electrical and Computer Engineering, Sultan Qaboos University, Muscat, Oman;

Email: hsoliman1@yahoo.com 
$G_{P S S}:$ Transfer function of PSS.

\section{Introduction}

$\mathrm{P}$ ower systems have to provide good service to consumers in terms of constant value of voltage and frequency. Automatic voltage regulators (AVR) are installed to Synchronous generators to adjust its terminal voltage. For tight control of terminal voltage, the AVR uses high gain in the loop (which may result in instability). Power systems are subject to small disturbances as a result of line switching, changing on the value of the load and other reasons. These small disturbances that happen on the system may grow to cause system separation; consequently, a great loss on the national economy. In order to decrease the swings that happen during transient rotor angle instability and fix the voltage value to constant value, a stabilizing signal is added to the excitation system. The power system stabilizers are developed to support and help in damping these low-frequency oscillations (1-3 Hz) by adding a signal to the excitation system ${ }^{[1-5]}$.

For PSS tuning, different techniques such as classical ${ }^{[6,7]}$, intelligent ${ }^{[8]}$, adaptive ${ }^{[9]}$, robust ${ }^{[10-15]}$, switching control ${ }^{[16]}$ methods are reported. However, considering the variable nature of the loads (causing system uncertainty), the robust control is the best choice. The main advantages of robust approach in comparison of other methods is in its simplicity (one controller with fixed parameters facing the system uncertainty due to load changes).

In the present paper, a robust control method based on Kharitonov's theorem is proposed to design a robust conventional PSS which guarantees satisfactory operation under uncertain operating conditions. In addition to not violating the practical ranges of the controller parameters. The Kharitonov's theorem addresses few polynomials which are obtained from closed loop characteristic equation. Unlike ${ }^{[12]}$ which uses the root locus method to stabilize many polynomials and does not consider the practical limits of the controller parameters, the suggested design avoids these difficulties by solving the problem using constrained nonlinear optimization approach.

This paper is organized as follows: the case study system, its structure and mathematical model for performance evaluation of the proposed robust control method , in sections 2 and 3, are described. In section 4, control design strategy based on Kharitonov's theorem, is presented. Simulation results and conclusion are presented in sections 5 and 6 respectively.

\section{Case Study System}

The case study system is Barka II power station. It is composed of $3 \times 200$ MVA steam $+2 \times 175$ MVA gas turbines, Figure 1a. The design of PSS installed at one of the steam turbines is presented in ${ }^{[18]}$. In that study, the problem is solved as a single machine infinite bus (SMIB) system. In which, the rest of the system (the other 4 machines+ the oman grid) is represented by Thevenin's equivalent using the short circuit fault level at the $11 \mathrm{kv}$ bus. This is shown in Figure $1 \mathrm{~b}$ by $V_{\infty}, x_{e}$. The PSS of ${ }^{[18]}$ is based on the heaviest load in summer and it has the notch filter structure to eliminate the conjugate complex poles near the imaginary axis of the system.

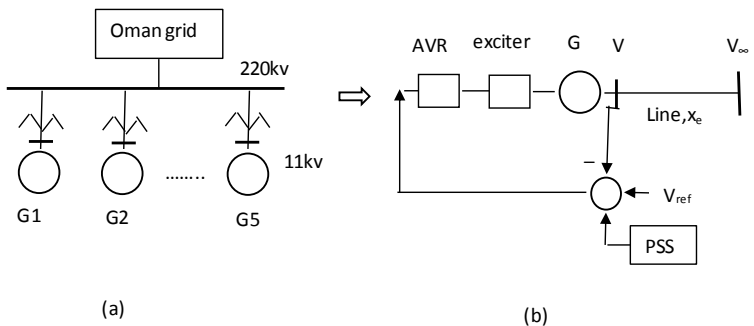

Figure 1 The case study system, Barka II power station

In present manuscript, a conventional PSS is designed for one of the gas turbines which achieves: (1) robustness against load variations, and (2) practical ranges of controller parameters.

\section{Mathematical Model}

The proposed robust saturated controller is tested using a single machine infinite bus system (SMIB). The mathematical nonlinear model describing the dynamics of SMIB is given by:

$$
\begin{aligned}
& \dot{\delta}=\omega_{0}(\omega-1) \\
& 2 H \dot{\omega}=P_{m}-P_{e}=P_{m}-\left[E_{q}^{\prime}+\left(x_{q}-x_{d}^{\prime}\right) I_{d}\right] I_{q} \\
& T_{d 0}^{\prime} \dot{E}_{q}^{\prime}=E_{q}-\left[E_{q}^{\prime}+\left(x_{d}-x_{d}^{\prime}\right) I_{d}\right] \\
& T_{E} \dot{E}_{f}=-E_{f}+K_{E}\left(V_{r e f}-V_{t}+u\right)
\end{aligned}
$$

where $I_{d}, I_{q}$, and $V_{d}$ are given by, Figure2:

$$
\begin{aligned}
& I_{d}=\frac{E_{q}^{\prime}-V \cos \delta}{x_{d}^{\prime}+x_{e}} \\
& I_{q}=\frac{V \sin \delta}{x_{q}+x_{e}} \\
& V=\sqrt{[}\left[\left(I_{q} x_{q}\right)^{2}+\left(E_{q}^{\prime}-I_{d} x_{d}^{\prime}\right)^{2]}\right.
\end{aligned}
$$




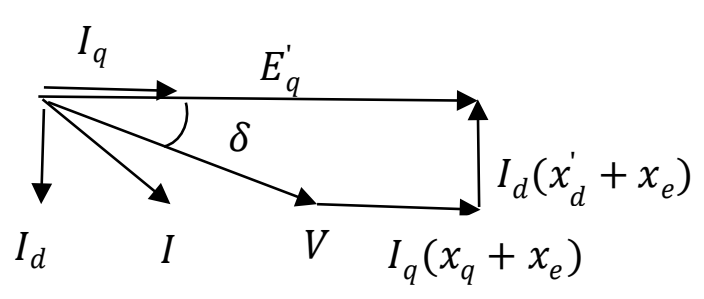

Figure 2. Phasor diagram

The SMIB data are in per unit (p.u.) unless otherwise stated and have their usual meaning ${ }^{[1]}$ and are listed in Table 1. Three operating conditions (light, nominal, and heavy) are considered to cover the whole range of load changes all over the year.

Table 1. Data for Barka power station II, gas turbine

\begin{tabular}{|c|c|}
\hline$x_{d}$ & 2.11 \\
\hline$x_{d}{ }^{\prime}$ & 0.207 \\
\hline$x_{q}$ & 1.97 \\
\hline$\omega_{o}$ & $314 \mathrm{rad} / \mathrm{s}$ \\
\hline$T_{d o}{ }^{\prime}$ & $10.8 \mathrm{~s}$ \\
\hline$H$ & $1.256 \mathrm{~s}$ \\
\hline$K_{E}$ & 25 \\
\hline$T_{E}$ & $0.05 \mathrm{~s}$ \\
\hline$x_{e}$ & 0.15 \\
\hline $\mathrm{P}_{\text {Light }}$ & 0.4 \\
$\mathrm{P}_{\text {Nominal }}$ & 0.7 \\
$\mathrm{P}_{\text {Heary }}$ & 1 \\
\hline $\mathrm{Q}_{\text {Light }}$ & 0.35 \\
$\mathrm{Q}_{\text {Nominal }}$ & 0.525 \\
$\mathrm{Q}_{\text {Heary }}$ & 0.875 \\
\hline $\mathrm{V}$ & 1 \\
\hline
\end{tabular}

Note that $H$ in the above table is calculated as follows:

$$
H=\text { kinetic energy / machine rating }=\frac{1}{2} J w^{2} / S
$$
$=0.5 * 5091 \mathrm{~kg} \cdot \mathrm{m}^{2} *(3000 \mathrm{rpm} \times 2 \pi \mathrm{f} / 60)^{2} /\left(175 \mathrm{MVA}^{*} 10^{6}\right)$

Substituting (2) into (1), and linearizing the resulting mathematical model for small disturbance around the three operating conditions (using the matlab commands trim, and linmod) to get the state equation:

$x=A x+B u, y=C x$

Where $x=\left[\Delta \delta, \Delta \omega, \Delta E_{q}^{\prime}, \Delta E_{f}\right]^{T}$ and the entries of the matrix $A$ are functions of the active and reactive machine load $(P, Q)$. The input $u$ is generated by the PSS. Since the most commonly used input to the PSS is the change in speed $\Delta \omega$, the matrix $C$ is selected as

$\mathrm{C}=[0,1,0,0]$

The plant (synchronous machine) transfer function model for the three operating conditions are

$$
\begin{aligned}
& G_{\text {Light }}=\frac{21.71 s}{s^{4}+20.22 s^{3}+233.8 s^{2}+4247 s+3900} \\
& G_{\text {Nominal }}=\frac{28.87 s}{s^{4}+20.22 s^{3}+326.3 s^{2}+6115 s+5511} \\
& G_{\text {Heavy }}=\frac{30.18 s}{s^{4}+20.22 s^{3}+425.3 s^{2}+8108 s+8056}
\end{aligned}
$$

By using the transfer functions of the nominal, heavy, and light loads, the plant uncertain transfer function is obtained by taking the minimum and the maximum coefficients value of the parameters.

$G_{\text {plant }}=\frac{[21.7130 .18] s}{s^{4}+20.22 s^{3}+[233.8425 .3] s^{2}+[42478108] s+[39008056\rceil}$

\section{Control system strategy}

The control target is to design a conventional PSS to robustly stabilize the uncertain plant (5) i.e. to damp the system oscillations in less than $10-15 \mathrm{sec}$ and not to violate the controller's parameters limitation imposed in industry. The conventional PSS can be a single lead or double lead controller

$$
\begin{aligned}
& G_{P S S, \text { Single lead }}=K \frac{T_{1} s+1}{T_{2} s+1} \\
& G_{P S S, \text { Double lead }}=K \frac{\left[T_{1} s+1\right]}{\left\lceil T_{2} s+1\right\rceil} \frac{\left[T_{3} s+1\right]}{\left\lceil T_{4} s+1\right\rceil}
\end{aligned}
$$

The practical ranges of PSS parameters (all positive scalars) are ${ }^{[3]}$ :

$0.1 \leq K \leq 50,0.2 \leq T_{1} \leq 1.5 \mathrm{sec}, 0.02 \leq T_{2} \leq 0.15 \mathrm{sec}$, $0.2 \leq T_{3} \leq 1.5 \mathrm{sec}, 0.02 \leq T_{4} \leq 0.15 \mathrm{sec}$.

The closed loop system is shown in Figure 3

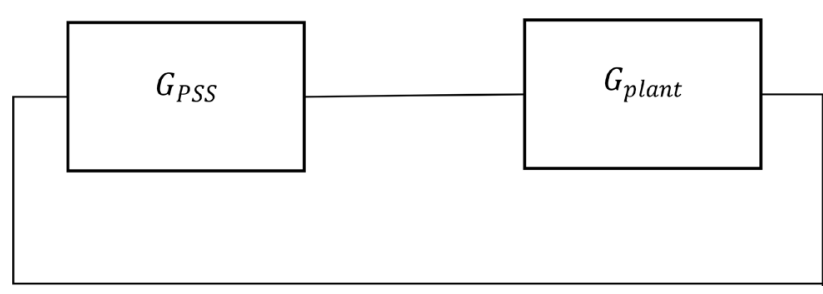

Figure 3. The closed loop system

The closed loop characteristic polynomial is

$1+G_{P S S} G_{\text {plant }}=0$

Note that (7) is an uncertain polynomial function of the PSS parameters.

\subsection{Kharitonov's Theorem}

When the coefficients of the characteristic polynomial are fixed, the best method is to use the Routh-Hurwitz to test 
if the system is stable or not. When the exact value parameters of the system are not fixed due to changing loads of the SMIB, an infinite number of polynomials have to be checked for stability using the Routh method. Kharitonov's theorem avoid such difficulty by checking the stability only 4 polynomials ${ }^{[17]}$. It can be used to estimate the stability of uncertain dynamical systems. When the range of the polynomial coefficients is known, the Kharitonov's theorem can be used to find the stability of the dynamical system. The polynomial

$p(s)=a_{n} s^{n}+a_{n-1} s^{n-1}+\ldots+a_{0}$

is called an interval polynomial when the coefficients $a_{i}$ is independent of each other and changes in an interval having minimum and maximum limit. An interval polynomial is represented as

$p(s)=\sum_{i=0}^{n}\left[a_{i}^{-}, a_{i}^{+}\right] s^{i}$

The system is robustly stable if and only if the following four Kharitonov polynomials are stable ${ }^{[17]}$.

$$
\begin{aligned}
& p_{1}=a_{0}^{-}+a_{1}^{-} s+a_{2}^{+} s^{2}+a_{3}^{+} s^{3}+a_{4}^{-} s^{4}+\ldots \\
& p_{2}=a_{0}^{+}+a_{1}^{+} s+a_{2}^{-} s^{2}+a_{3}^{-} s^{3}+a_{4}^{+} s^{4}+\ldots \\
& p_{3}=a_{0}^{+}+a_{1}^{-} s+a_{2}^{-} s^{2}+a_{3}^{+} s^{3}+a_{4}^{+} s^{4}+\ldots \\
& p_{4}=a_{0}^{-}+a_{1}^{+} s+a_{2}^{+} s^{2}+a_{3}^{-} s^{3}+a_{4}^{-} s^{4}+\ldots
\end{aligned}
$$

Note that (9) is a polynomial of descending power of s. Whereas, (10-13) are polynomials of ascending powers of s. The "-" and "+" show the minimum and maximum bounds of the polynomial coefficients. In the Kharitonov's theorem, the $p(\mathrm{~s})$ is considered as the closed loop polynomial. In our PSS problem, the polynomial coefficients are dependent. In this case, the Kharitonov's theorem represents only a sufficient condition for the stability of uncertain polynomial if the coefficients are assumed independent. Kharitonov's theorem provides a simple and powerful tool for robust adjusting of practical controllers.

\subsection{Application of Kharitonov's Theorem to Ro- bust PSS Design}

It is very difficult to try to find an analytical expression for the closed loop uncertain polynomial as a function of the PSS parameters (all are positive scalars), extracting the 4 Kharitonov's polynomials, arranged an ascending powers of s. This can be easily done numerically using the matlab commands: conv, flip. Trying to stabilize the Kharitonov's polynomials by adjusting the PSS parameters using the root locus method as presented in ${ }^{[12]}$ is also difficult. The easiest way is to cast the robust PSS problem in the following constrained nonlinear optimization to shift the rightmost closed loop pole (among the 4 kharitonov's polynomials) to the left in the complex plane as much as possible.

minimize $_{K, T 1 . T 4} J=\max \{$ real part of closed loop pole $\} \forall p_{1} . p_{4}$

Subject to the following constraints

$0.1 \leq K \leq 50,0.2 \leq T_{1} \leq 1.5,0.02 \leq T_{2} \leq 0.15,0.2 \leq T_{3} \leq 1.5$, $0.02 \leq T_{4} \leq 0.15$.

The optimization problem (14) is highly nonlinear. To avoid trapping into a local minimum solution, it is best solved using a probabilistic approach e.g. the particle swarm optimization (matlab command, "particleswarm").

Solving (14), the obtained single lead and double lead robust PSS (providing the max possible left shift to the rightmost closed loop poles, thus achieving the fastest oscillation damping) are

$$
\begin{aligned}
G_{\text {Single lead }} & =13.0602 \frac{1.5 s+1}{0.02 s+1} \\
G_{\text {Double lead }} & =1.8635 \frac{[1.5 s+1]}{[0.02 s+1]} \frac{[1.5 s+1]}{[0.02 s+1]}
\end{aligned}
$$

As seen there is no violation to the practical ranges of the PSS parameters.

\section{Performance Evaluation}

For a cleared three phase short circuit fault on the generator terminal which causes $0.1 \mathrm{rad}$ angle disturbance, the performance at different loads is given in Figure 4,a,b,c, for single lead robust PSS.

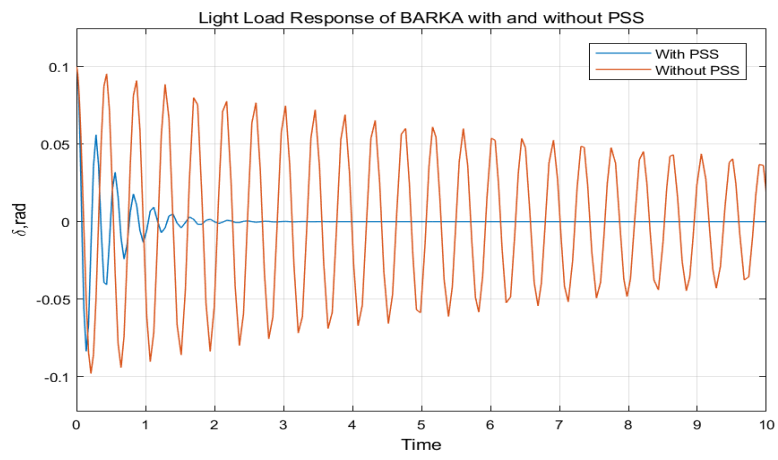

Figure 4 (a). Light Load Response of Barka Power II station with and without PSS 


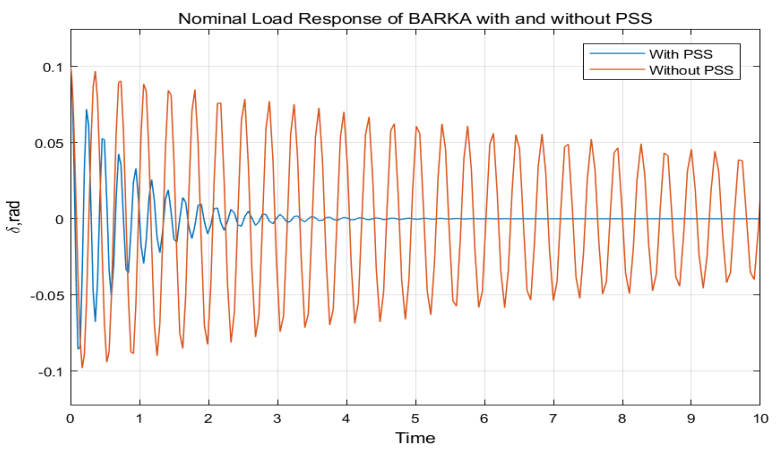

Figure 4 (b). Nominal Load Response of Barka Power II station with and without PSS

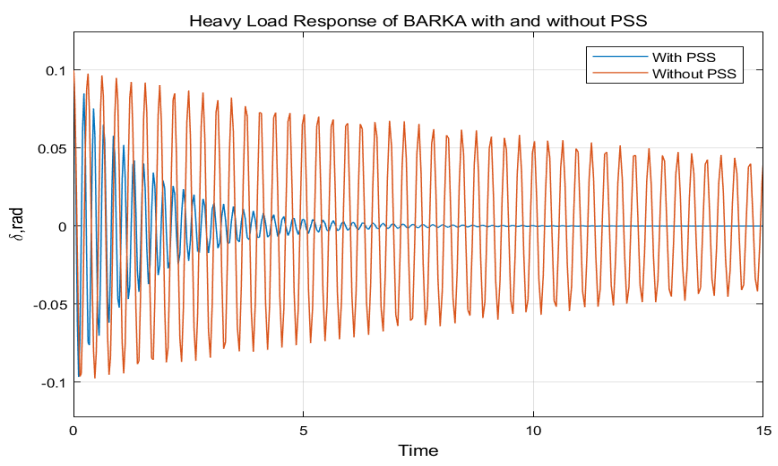

Figure 4 (c). Heavy Load Response of Barka Power II station with and without PSS

Similarly for double lead robust PSS, the response at different loads is shown in Figure 5a,b,c.

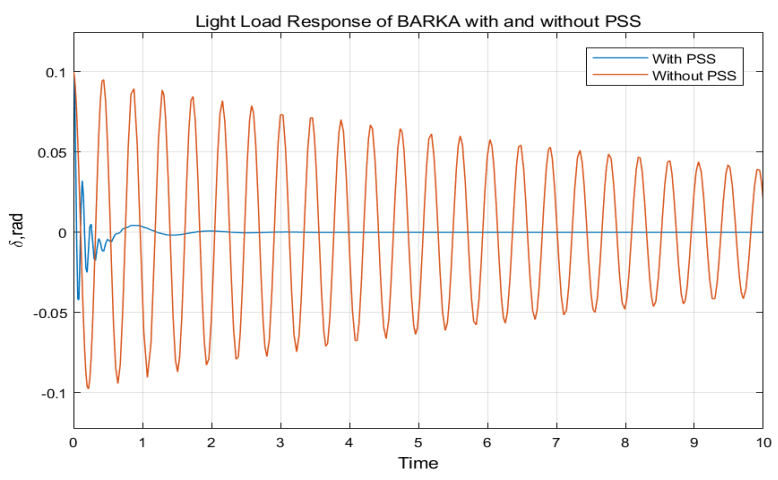

Figure 5 (a). Light Load Response of Barka Power II station with and without PSS

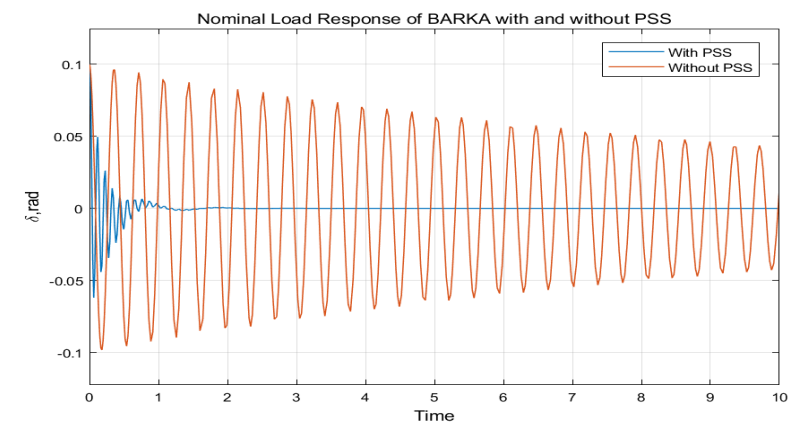

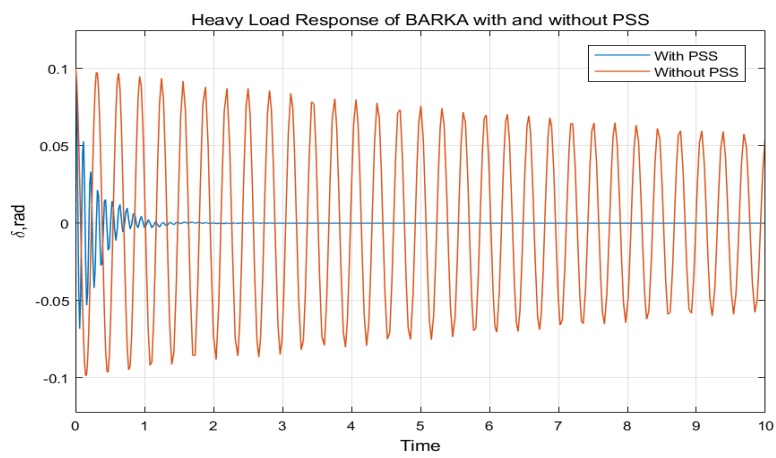

Figure 5 (b) (c). Heavy Load Response of Barka Power II station with and without PSS

It is evident that when the load increases (the system becomes nearer to instability), the settling time increases.

Out of Figure 4,5, the following settling time comparison can be done between the single lead and double lead robust PSS, Table 2 .

Table 2. Settling time, s

\begin{tabular}{|c|c|c|}
\hline & Single lead robust PSS & Double lead robust PSS \\
\hline Light load & 3 & 2 \\
\hline Nominal load & 5 & 2.1 \\
\hline Heavy load & 10 & 2.5 \\
\hline
\end{tabular}

If the designer is not satisfied with a single lead PSS at heavy load (the settling time is $10 \mathrm{~s}$ ), he can use the double lead PSS. The price is more complex hardware.

\section{Conclusion}

A simple method is proposed for the design of the PSS. The method is based on Kharitonov's theorem. The suggested design retains the conventional PSS structure with controller parameters in the practical ranges. Robustness of the proposed controller is evaluated via several simulations in the MATLAB/SIMULINK environment. The double lead robust PSS is more effective in quenching system oscillations than the single lead. The price is more hardware complexity.

\section{References}

[1] Prabha Kundur. Power System Stability and Control, MCGRAW-HILL, 2005.

[2] Jan Machowski, Janusz Bialek, Jim Bumby. Power System Dynamics: Stability and Control, 2e, John Wiley \& Sons Inc, New York, United States, 2008.

[3] Peter W. Sauer, M. A. Pai, Joe H. Chow. Power System Dynamics and Stability: With Synchro-phasor Measurement and Power System Toolbox, 2e, John Wiley and Sons Ltd, Hoboken, United States, 2018. 
[4] Lingling Fan. Control and Dynamics in Power Systems and Microgrids, CRC Press, 2017.

[5] P.M. Anderson, A.A. Fouad. Power System Control and Stability. Iowa State University Press, Ames, IA, 1977.

[6] F.P. DeMello and C. Concordia. Concepts of Synchronous Machine Stability as Affected by Excitation Control. IEEETrans., PAS-88, 1969: 316-329.

[7] W.G.Heffron and R.A.Phillips. Effects of Modern Amplidyne Voltage Regulator in Under-excited Operation of Large Turbine Generators. AIEETrans., PAS-71, 1952: 692-697.

[8] Hassan Yousef, Hisham Soliman, Mohamed Albadi , Nonlinear Power System Excitation Control Using Adaptive Wavelet Networks, Neurocomputing, 2017, 230: 302-311.

[9] K. Tang and G. K. Venayagamoorthy. Adaptive inter-area oscillation damping controller for multi-machine power systems. Electric Power Systems Research, 2016, 134: 105- 113 .

[10] Hisham M. Soliman , Hassan A. Yousef, Rashid AlAbri, and Khaled A. El-Metwally, Decentralized Robust Saturated Control of Power Systems Using Reachable Sets, , Wiley-Hindawi Complexity, Volume 2018, Article ID 2563834, 12 pages. https://doi.org/10.1155/2018/2563834

[11] B. Pal and B. Chaudhuri, Robust Control in Power Systems, Springer, 1st edition, 2005.
[12] H. M. Soliman, A. L. Elshafei, A. A. Shaltout, and M. F. Morsi. Robust power system stabilizer. IEE Proceedings - Generation, Transmission and Distribution, 2000, 147(5): 285- 291.

[13] H. M. Soliman. Resilient observer-based power system stabilizers. Transactions of the Institute of Measurement and Control, 2016, 38(8): 981-991.

[14] H. M. Soliman and H. A. Yousef. Saturated robust power system stabilizers. International Journal of Electrical Power \& Energy Systems, 2015, 73: 608614.

[15] H.M. Soliman and K. El-Metwally. Robust pole placement for power systems using two-dimensional membership fuzzy constrained controllers. IET Generation, Transmission \& Distribution, 2017, 11(16): 3966-3973.

[16] H.M. Soliman and M. Shafiq. Robust stabilisation of power systems with random abrupt changes. IET Generation, Transmission \& Distribution, 2015, 9(15): $2159-2166$.

[17] S.P. Bhattacharyya, A. Datta, L.K. Keel. Linear control theory: structure, robustness, and optimization. CRC 2009.

[18] H. M. Soliman , M. H. Albadi, A. Al-Maamari, and A. Al-Kasbi. Power System Stabilizer Design using Notch-filter - Barka II Case Study. to appear in The Journal of Engineering Research [TJER], 2019, 16(2): 87-95. 